(2) Open Access Full Text Article

ORIGINAL RESEARCH

\title{
Hongjingtian Injection Inhibits Proliferation and Migration and Promotes Apoptosis in High Glucose-Induced Vascular Smooth Muscle Cells
}

This article was published in the following Dove Press journal:

Drug Design, Development and Therapy

\author{
Zhengyuan Fan $^{1,2}$ \\ Congcong Guo ${ }^{3}$ \\ Yuhan Zhang 2,4 \\ Jinming $\mathrm{Yao}^{2,4}$ \\ Lin Liao ${ }^{2,4}$ \\ Jianjun Dong ${ }^{5}$
}

'First Clinical Medical College, Shandong University of Traditional Chinese

Medicine, Jinan 250355, People's Republic of China; ${ }^{2}$ Division of Endocrinology,

Department of Internal Medicine,

Shandong Provincial Qianfoshan Hospital,

Shandong University, Jinan 2500 I4,

People's Republic of China; ${ }^{3}$ Division of

Endocrinology, Department of Internal

Medicine, Affiliated Hospital of Shandong

University of Traditional Chinese

Medicine, Jinan 2500I I, People's Republic

of China; ${ }^{4}$ Division of Endocrinology,

Department of Internal Medicine, The

First Affiliated Hospital of Shandong First

Medical University, Jinan 2500 I4, People's

Republic of China; ${ }^{5}$ Division of

Endocrinology, Department of Internal

Medicine, Qilu Hospital of Shandong

University, Jinan 2500I2, People's

Republic of China
Correspondence: Lin Liao; Jianjun Dong Division of Endocrinology, Department of Internal Medicine, Shandong Provincial

Qianfoshan Hospital, Shandong

University, Jinan 2500I4, People's

Republic of China

Tel +86-15168888260;

+86- |379| I 22910

Email liaolin@sdu.edu.cn;

cwc_II@sdu.edu.cn
Background: Hongjingtian injection (HJT) is administered in the treatment of vascular diseases, including diabetic angiopathies (DA). However, its underlying mechanisms have not been examined systematically.

Methods: In this research, we explored potential mechanisms of HJT through network pharmacology. HG-stimulated A7r5 cells served as the cell model. Cell proliferation, migration and apoptosis were investigated. The effects on key targets and the AKT pathway were verified by Western blotting in experiments with the AKT inhibitor LY294002 or activator SC79.

Results: Network analysis predicted that HJT targeted 10 candidate targets and 15 pathways including cell proliferation, migration and apoptosis in response to DA. Functional experiments showed that HJT markedly suppressed the proliferation and migration and promoted the apoptosis of HG-induced VSMCs, which validated the prediction. Mechanistically, HJT significantly downregulated the expression of pAKT, MMP9, and PCNA, upregulated the expression of $\mathrm{p} 53$ and cleaved caspase- 3 and increased the Bax/Bcl-2 ratio compared with the HG group. SC79, an AKT activator, partially reversed the inhibitory effects of HJT on HG-induced VSMCs, confirming the involvement of the AKT pathway. Furthermore, the presence of the AKT inhibitor LY294002 had a similar inhibitory effect as HJT.

Conclusion: These findings systematically evaluate the potential mechanisms of HJT for the treatment of DA. HJT suppressed the proliferation and migration and promoted the apoptosis of HG-induced VSMCs partly by inhibiting the AKT pathway. Additionally, this study may provide a quick and effective way to investigate the molecular mechanisms of traditional Chinese medicine.

Keywords: network pharmacology, diabetic angiopathies, traditional Chinese medicine, diabetes

\section{Introduction}

The incidence of diabetes mellitus (DM) is rapidly increasing worldwide. ${ }^{1}$ Diabetic angiopathies (DA), particularly atherosclerosis (AS), are the leading causes of disability and mortality in this large cohort of patients. ${ }^{2}$ Although the mechanisms of DA are still incompletely understood, risk factors converge on the vascular wall, specifically vascular smooth muscle cells (VSMCs). ${ }^{3}$ Hyperglycemia facilitates the proliferation and migration of $\mathrm{VSMCs}^{4}$ and inhibits the apoptosis of VSMCs. ${ }^{5}$ VSMC dysfunction promotes the growth of plaque volume, accelerates the deposition of extracellular matrix (ECM) and ultimately leads to vascular remodeling. ${ }^{6}$ Therefore, targeting VSMCs might be a new option for the treatment of DA. 
Hongjingtian injection (HJT) is manufactured from water-soluble extracts of Rhodiola wallichiana, and has been used to treat vascular diseases for many years in China. Clinical studies have shown that HJT improves hemorheology, reduces intima-media thickness and relieves atherosclerotic inflammatory reactions., ${ }^{7,8}$ However, the mechanism of HJT in the clinical application for DA therapy is not well understood. Due to its multicomponent, multitarget properties, it is challenging to elucidate the pharmacological effects of HJT. Fortunately, network pharmacology, which is based on systems biology and computer technology, is emerging as an efficient tool to illuminate complex interactions between drugs and biological systems. ${ }^{9}$

In this study, both integrative network analysis and experimental verification were used. First, the potential targets of HJT were predicted by screening various databases. Then the candidate targets and potential mechanisms of HJT in the treatment of DA were analyzed. Ultimately, cell experiments were employed to verify the network analysis results (Figure 1).

\section{Materials and Methods}

\section{Chemicals and Reagents}

HJT was purchased from Tonghua Yusheng Pharmaceutical Co. Ltd. (Tonghua, China), which is a standardized medical product approved by the Sino Food and Drug Administration (SFDA) (Z20026866). HJT mainly consists of five ingredients: gallic acid, p-hydroxybenzoic acid, tyrosol, salidroside and p-hydroxycinnamic acid. ${ }^{10,11}$ SC79 and LY294002 were purchased from MedChem Express (Monmouth Junction, USA). The concentration of SC79 was $2.5 \mu \mathrm{M}^{12}$ and the concentration of LY294002 was $10 \mu \mathrm{M} .{ }^{13}$

\section{Screening for the Potential Therapeutic Targets of HJT in Diabetic Angiopathies}

The corresponding targets of the five ingredients were obtained from three validated target databases, and three predicted target databases. They were (1) PubChem (http://pubchem.ncbi.nlm. nih.gov), (2) Binding Database (https://www. bindingdb.org/ bind/index.jsp), (3) Traditional Chinese Medicine Systems Pharmacology Database (TCMSP, http://lsp.nwu.edu.cn/ tcmsp.php), (4) Similarity Ensemble Approach(SEA, http:// sea.bkslab.org/), (5) STITCH (http://stitch.embl.de/) and (6) Swiss Target Prediction (http://www.swisstargetpredic tion.ch/). The genes associated with DA were obtained from the Comparative Toxicogenomics Database (CTD, http:// ctdbase.org/). Only the Homo sapiens genes with the top 50 reference counts were chosen. Then, these genes were compared to the HJT-relevant genes to obtain the overlapping genes of HJT in the treatment of DA.

\section{Network Construction}

The following three visualized networks were constructed with Cytoscape 3.2.1.:

1. Drug-targets network (D-T network). The five components of HJT and their relevant targets generated the D-T network.

2. Drug- candidate targets network (D-C network). The five ingredients of HJT and their candidate targets in the treatment of DA were included in the D-C network.

3. Protein-protein interaction network (PPI network). The correlated proteins of candidate targets were obtained from the String database (http://string-db. org/). All these targets formed the PPI network. The topological property of the PPI network was analyzed and the targets with a degree greater than the average degree (6.83) were carried for further functional analysis.

\section{Functional Analysis}

The functional annotations of the critical targets were investigated by Database for Annotation, Visualization and Integrated Discovery (DAVID, http://david.abcc. ncifcrf.gov/). The cutoff criterion was set to a $p$-value $<0.01$. Additionally, the top 15 biological processes meeting the criterion were visualized by bubble charts.

\section{Cell Culture and Treatments}

A7r5 cells, ${ }^{14}$ a rat thoracic aorta smooth muscle cell line, were obtained from Procell (Wuhan, China). The cells were cultured in Dulbecco's modified Eagle's medium (DMEM) with $10 \%$ fetal bovine serum (FBS) and $1 \%$ penicillin-streptomycin (all from Gibco, NY, USA). All cells were maintained in $5 \% \quad \mathrm{CO}_{2}$ at $37^{\circ} \mathrm{C}$ under a humidified atmosphere. After serum starvation for $24 \mathrm{hrs}$, cells were exposed to DMEM containing 5.5 $\mathrm{mmol} / \mathrm{L} \mathrm{D}$-glucose $+19.5 \mathrm{mmol} / \mathrm{L}$ D-mannitol (MG), 25 $\mathrm{mmol} / \mathrm{L} \mathrm{D}$-glucose (HG), $25 \mathrm{mmol} / \mathrm{L} \mathrm{D}$ - glucose $+10 \mathrm{~mL} /$ $\mathrm{L}$ HJT(HG + HJT10), $25 \mathrm{mmol} / \mathrm{L}$ D- glucose $+20 \mathrm{~mL} / \mathrm{L}$ HJT(HG + HJT20), or $25 \mathrm{mmol} / \mathrm{L}$ D- glucose $+40 \mathrm{~mL} / \mathrm{L}$ HJT (HG + HJT40). ${ }^{15}$ For the inhibition experiments, cells were divided into five groups: MG, HG, HG + LY294002, 


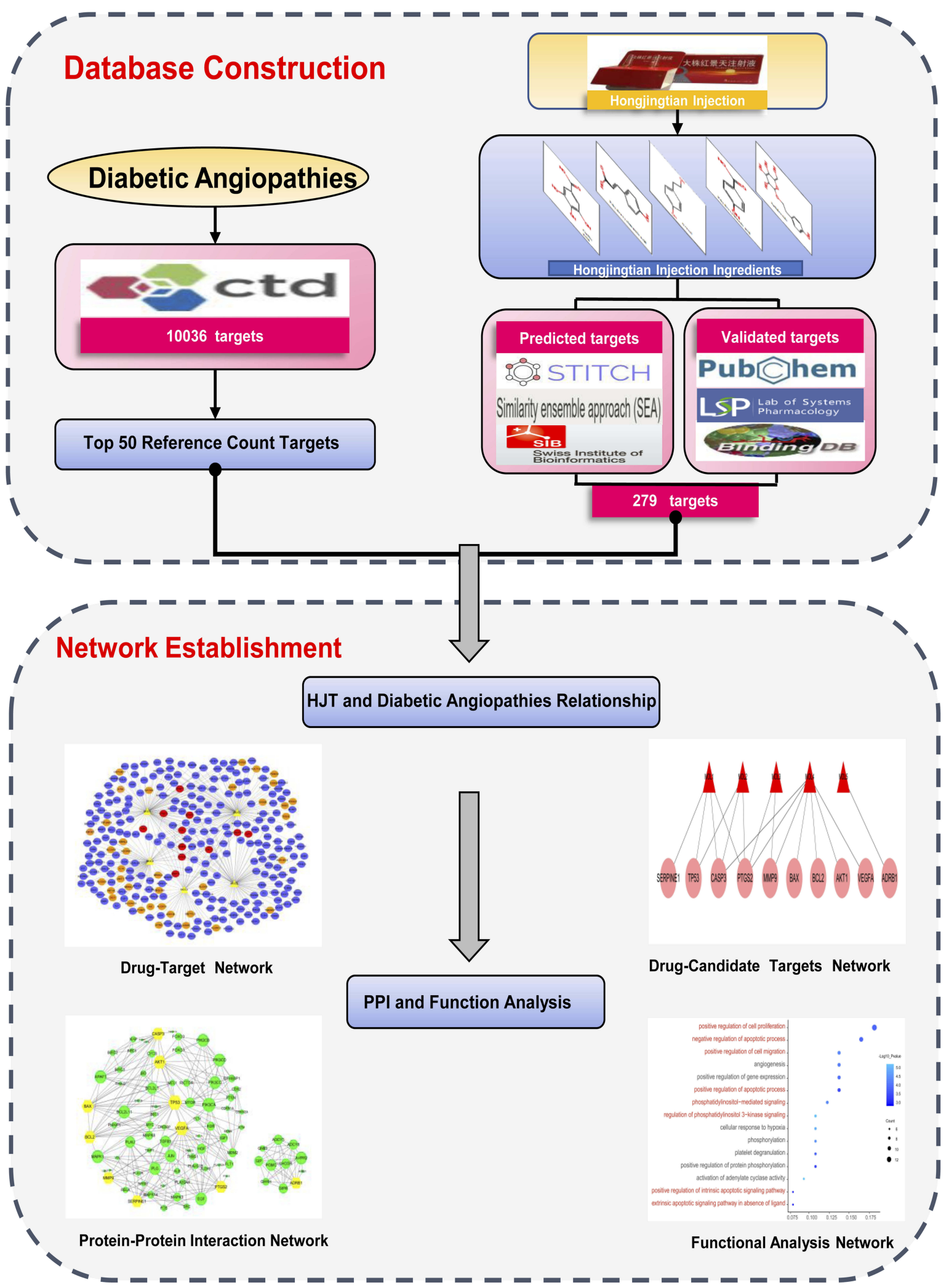

Figure I A schematic diagram. 
HG + HJT40, HG + HJT40 + LY294002. The cells were treated with $\mathrm{MG}, \mathrm{HG}, \mathrm{HG}+\mathrm{SC} 79, \mathrm{HG}+\mathrm{HJT} 40, \mathrm{HG}+$ HJT40 + SC79 for the activation experiments.

\section{Cell Viability and Proliferation Analysis}

The cytotoxicity of HJT and cell proliferation were evaluated by the Cell Counting Kit-8 assay (CCK-8) (Dojindo Molecular Technologies, Inc., Kumamoto, Japan). VSMCs $\left(1.5 \times 10^{3} /\right.$ well $)$ were cultured in 96 -well plates. First, cells were exposed to $\mathrm{MG}$ medium and treated with different concentrations of HJT $(10,20,40,80,160$, or $320 \mathrm{~mL} / \mathrm{L})$. After $48 \mathrm{hrs,}$, cell viability was analyzed by CCK8. Second, VSMCs were exposed to the conditions mentioned above (MG, HG, HG + HJT10, HG + HJT20, or $\mathrm{HG}+$ HJT40) for $48 \mathrm{hrs}$. Cell proliferation was evaluated by the CCK- 8 absorbance values.

\section{Wound Healing Assay}

VSMCs $\left(1.5 \times 10^{5} /\right.$ well $)$ were seeded in six-well plates. A linear wound was made with a $10 \mu \mathrm{L}$ pipette tip on the cell layer. PBS was used to remove the wounded cells. This time point was set as $0 \mathrm{hrs}$. VSMCs were then exposed to different treatments (MG, HG, HG + HJT10, HG + HJT20, or HG + HJT40) as indicated for 24, or $48 \mathrm{hrs}$. The scratch wound was imaged at 0,24 and $48 \mathrm{hrs}$ via microphotography. The wound healing rate was calculated by ImageJ. ${ }^{16}$

\section{Transwell Assay}

VSMCs were plated on six-well plates and treated with different media for $48 \mathrm{hrs}$. Then, the pretreated cells $(1 \times$ $10 \%$ chamber) were seeded into the upper chamber (Corning, NY, USA) with 0.5\% FBS DMEM. The lower chambers were filled with DMEM supplemented with $10 \%$ FBS. After incubation for six hours, VSMCs that had migrated to the lower chamber were stained by crystal violet. Cells were then counted in five randomly selected fields with a magnification of $200 \times$.

\section{Western Blotting}

Electrophoresis was used to separate the proteins of VSMC lysates. The proteins were transferred onto membranes and incubated overnight with the appropriate primary antibody. The secondary antibody was then used to bind and visualize the primary antibody. Mouse anti-Bcl-2 (Sc-7382) was obtained from Santa Cruz Biotechnology (Dallas, USA); rabbit anti-MMP9(Ep1254) was obtained from Abcam(Cambridge, UK); rabbit anti-cleaved caspase-3 (Asp175), rabbit anti-AKT (C67E7), rabbit antiphospho-AKT (Ser473), rabbit anti-PCNA (D3H8P) and mouse anti-p53(1c12) were obtained from Cell Signaling Technology (Danvers, MA, USA); and mouse anti-Bax (Gb11007) was obtained from Servicebio (Wuhan, China).

\section{Annexin V-FITC/Propidium lodide Assay}

Apoptosis was evaluated by an Annexin V-FITC/PI apoptosis assay kit (Neobioscience, Inc., Shenzhen, China). VSMCs were stimulated for $48 \mathrm{hrs}$, then adjusted to a density of $4.0 \times 10^{6}$ cells $/ \mathrm{mL}$ and resuspended in $195 \mu \mathrm{L}$ of binding buffer. Subsequently, the cells were stained with 5 $\mu \mathrm{L}$ of annexin V-FITC and $10 \mu \mathrm{L}$ of propidium iodide for 10 mins. The rate of positive cells was identified by flow cytometry.

\section{TUNEL Assay}

A TUNEL Apoptosis Assay Kit (Beyotime Biotechnology, Shanghai, China) was used to assess apoptotic cells. VSMCs were cultured on glass coverslips and pretreated for $48 \mathrm{hrs}$ as described above. Then, cells were subjected to the TUNEL assay and imaged (magnification, $\times 200$ ).

\section{Statistical Analysis}

Statistical analysis was conducted using SPSS 21.0 software. The results are expressed as the mean $\pm \mathrm{SD}$. Student's $t$-test was performed to analyze the difference between the experimental and control groups. The oneway analysis of variance (ANOVA) test followed Turkey's posttest was used to analyze the difference among multiple groups. $P$ value $<0.05$ was regarded as significant.

\section{Results}

\section{The Candidate Targets of HJT Against Diabetic Angiopathies}

First, we identified 279 targets of HJT through the six target fishing database (Table S1). As shown in Figure 2A, the D-T network consisted of 284 nodes and 414 interactions. Second, we searched the CTD database with the keyword "diabetic angiopathies" and extracted the top 50 genes with the highest reference counts (Table S2). Finally, the 50 genes were mapped to the 279 genes to obtain the 10 candidate genes. They were $V E G F A$, AKT1, PTGS2, CASP3, BCL2, SERPINE1, TP53, BAX, $A D R B 1$ and MMP9. Interestingly, salidroside, the main active compound of HJT, was associated with seven 

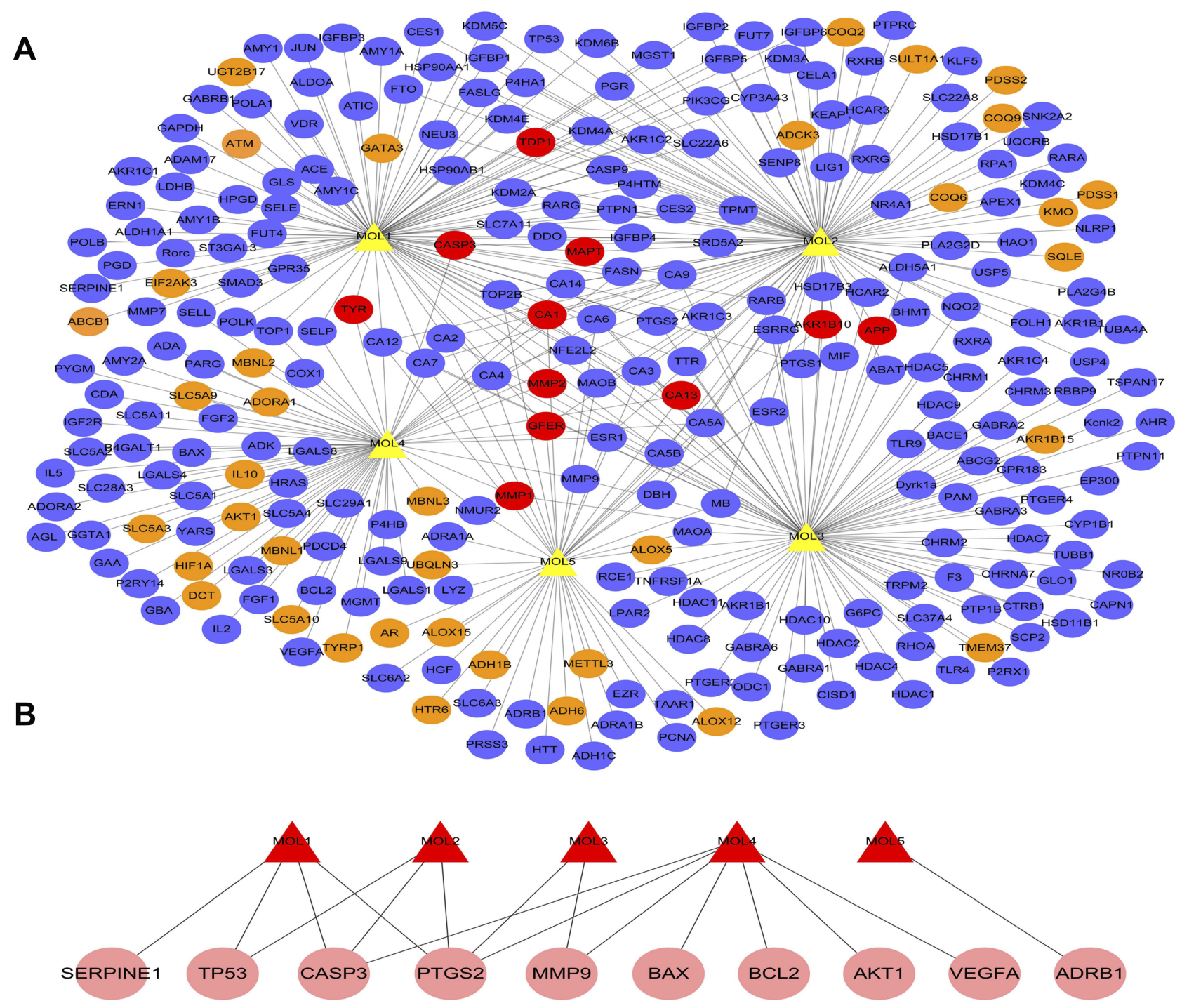

Figure 2 The drug-target network of HJT (A) and drug-candidate target network of HJT for treating DA (B). (A) The components of HJT are indicated by yellow triangles. The blue circles represent validated targets, the orange circles represent predicted targets and the red circles represent both validated and predicted targets. (B) The nodes in red refer to components of HJT, and the nodes in pink refer to candidate targets.

Abbreviations: MOLI, gallic acid; MOL2, p-hydroxybenzoic acid; MOL3, p-hydroxycinnamic acid; MOL4, salidroside; MOL5, tyrosol.

targets and might play an important role in DA treatment (Figure 2B).

\section{PPI Network and Functional Analysis}

Generating a PPI network has been used as an effective method to identify indirect targets. ${ }^{17}$ As illustrated in Figure 3A, the PPI network was composed of 304 interactions involving the 10 candidate targets and 79 indirect targets. The nodes with larger degree values are usually the crucial targets in this network. Ultimately, 38 targets with a degree value greater than the average degree were selected as the critical targets (Table S3).
To further annotate the targets, DAVID bioinformatics resources were used to analyze the biological process (BP) of the 38 crucial targets. Figure $3 \mathrm{~B}$ demonstrates the top 15 gene functions of the critical targets. The top gene function was related to cell proliferation, which had the highest degree (degree=12). In addition to the apoptotic process (degree $=11)$, cell migration (degree $=9$ ) and the phosphatidylinositol (PI)-mediated signaling pathway (degree $=8$ ) were regarded as the crucial biological processes of HJT in the regulation of DA. Interestingly, these processes all interacted with AKT, which implied that the AKT pathway might be the key pathway. 
A
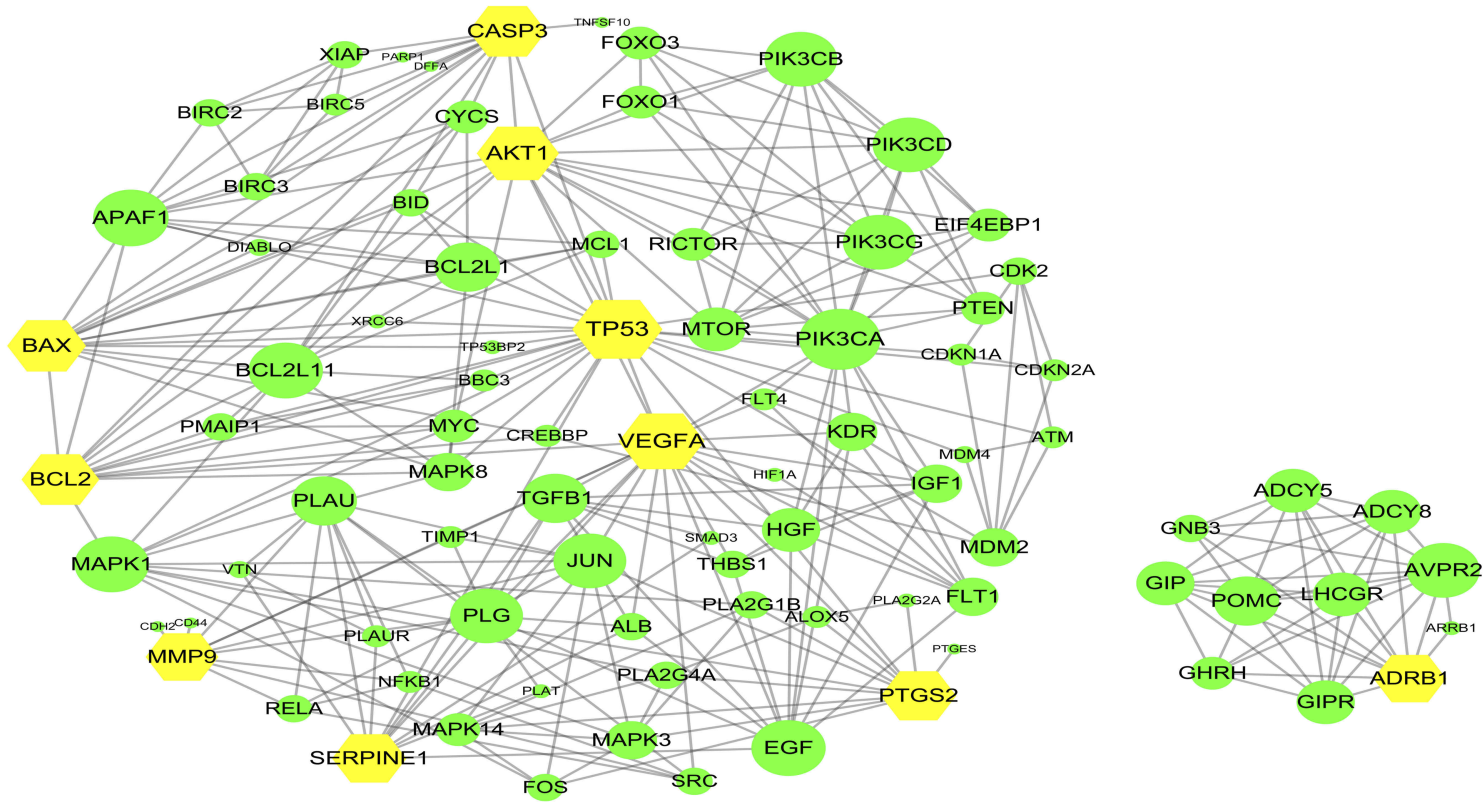

B

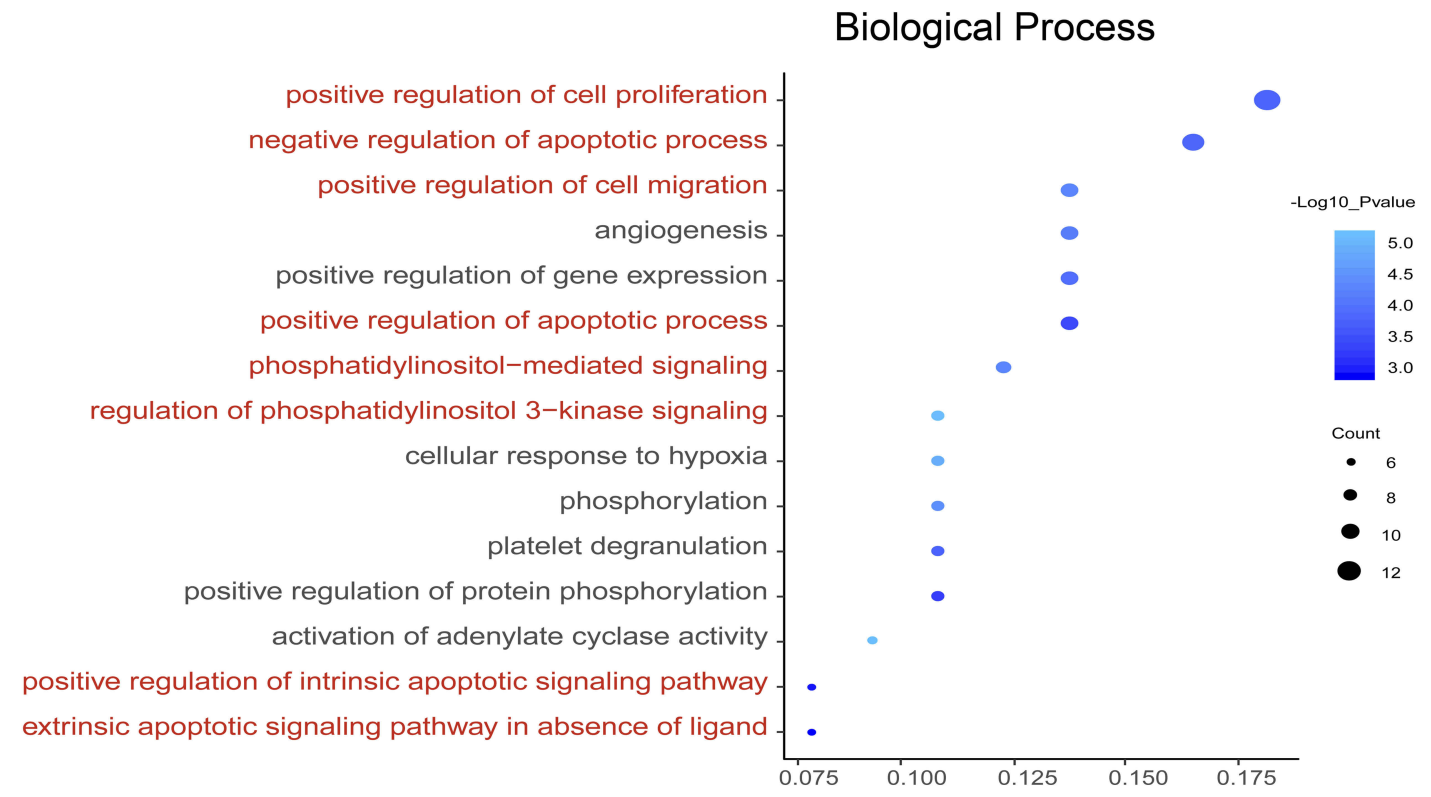

Figure 3 The protein-protein interaction network (A) and the top 15 functional analysis of the 38 crucial targets (B). The candidate targets are shown as yellow hexagons, and the corresponding proteins are represented by green. The node size of these targets corresponds to the degree value.

\section{The Concentration Determination of HJT} The suitable concentration of HJT was determined by CCK-8 assay. As shown in Figure 4A, cell viability did not change significantly within $48 \mathrm{hrs}$ in the range of $0-40 \mathrm{~mL} / \mathrm{L} \mathrm{HJT}$. At concentrations in excess of $40 \mathrm{~mL} / \mathrm{L}$, HJT obviously decreased cell survival. The half-maximal inhibitory concentration (IC50) of HJT for VSMCs was $83.66 \mathrm{~mL} / \mathrm{L}$. Thus, $40 \mathrm{~mL} / \mathrm{L}$ HJT, approximately half of the IC50 value, was selected as the high concentration and $20 \mathrm{~mL} / \mathrm{L}$ and $10 \mathrm{~mL} / \mathrm{L}$ HJT were selected as the medium and low concentrations, respectively, for subsequent experiments.

\section{HJT Significantly Inhibited the} Proliferation of VSMCs Induced by HG

The CCK-8 assay was also used to assess VSMC proliferation. As shown in Figure 4B, HG stimulated the proliferation of VSMCs compared with MG $(\mathrm{P}<0.001)$, and HJT substantially suppressed HG-induced VSMC proliferation ( $\mathrm{P}<0.05$ vs HG) in a dose-dependent manner. The results showed that HGinduced proliferation was significantly lower by approximately $50 \%$ after $40 \mathrm{~mL} / \mathrm{L}$ HJT stimulation. These data revealed that HJT could significantly suppress the HG-induced proliferation of VSMCs. 
A

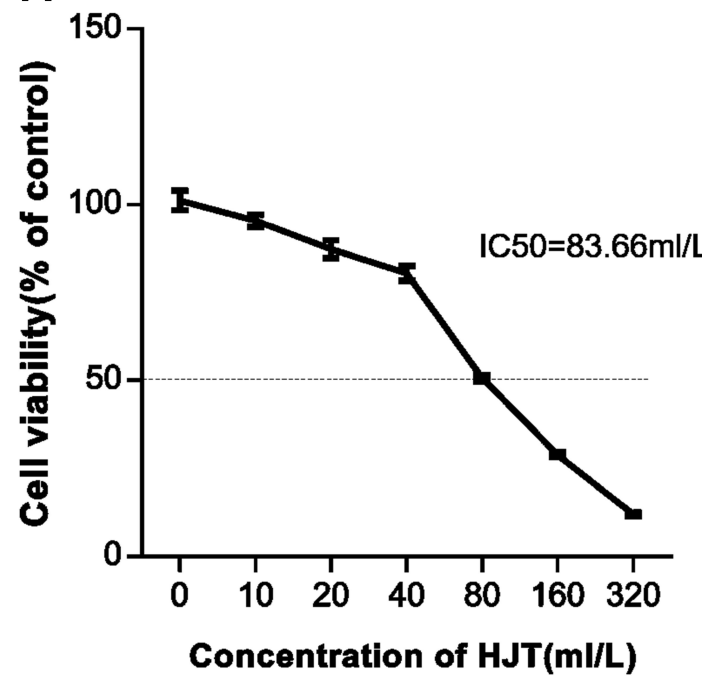

B

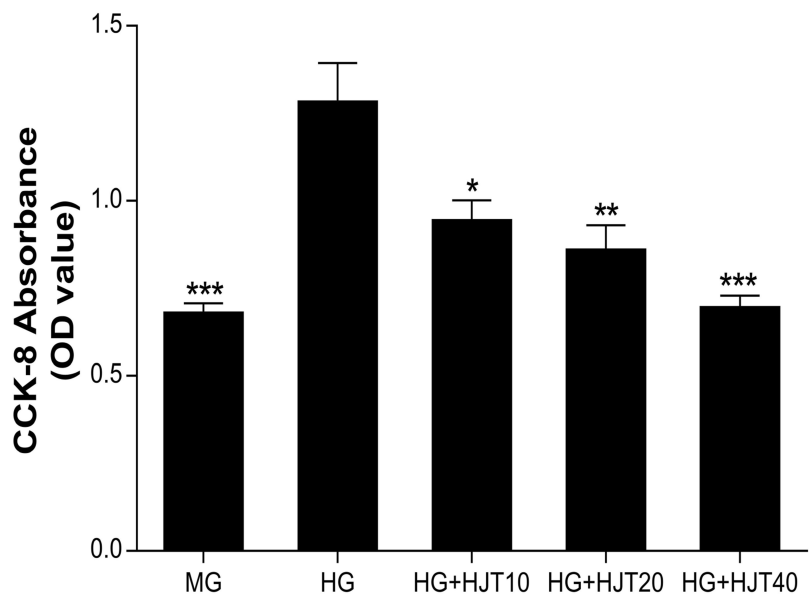

Figure 4 (A). Cell viability induced by different concentrations of HJT. (B). The antiproliferative effects of HJT in HG-induced VSMCs. Results were presented by mean \pm SD. $*_{\mathrm{p}}<0.05, * * \mathrm{p}<0.01, * * * \mathrm{p}<0.001$ all compared with $\mathrm{HG}$ group.

Abbreviations: IC50, half-maximal inhibitory concentration; CCK-8, Cell Counting Kit-8; OD, optical density; HJT, Hongjingtian Injection; MG, mannitol group; HG, high glucose; HJT I0, I0mI/L HJT; HJT 20, 20ml/L HJT; HJT 40, 40ml/L HJT.

\section{HJT Inhibited the Migration of VSMCs Induced by HG}

The Transwell assay and wound healing assay were performed to assess cell migration (Figure 5). As expected, VSMC migration was markedly increased by $\mathrm{HG}(P<0.001$ vs MG). However, HJT at concentrations of 20 and $40 \mathrm{~mL} / \mathrm{L}$ significantly abolished the increased migration of VSMC $(P<0.001$ vs HG). Using the wound healing assay, HG significantly enhanced the VSMC migration of two folds for 48 hrs compared with MG. HJT prevented these increases in cells exposed to HG. These results indicated that HG promoted VSMC migration, and that HJT exhibited effective inhibitory activity on the migration of VSMCs.

\section{HJT Reversed the Inhibitory Effect of HG on Apoptosis in VSMCs}

The Annexin V-FITC staining and TUNEL assay were used to study the effects of HJT on cell apoptosis (Figure 6). Decreased cell apoptosis was observed in the HG group compared with the MG group by the TUNEL assay. Treatment with HJT significantly increased VSMC apoptosis. In addition, HJT also significantly increased the Annexin V+/PIsubpopulation (early apoptotic cells) in VSMCs, whereas the Annexin $\mathrm{V}+/ \mathrm{PI}+$ subpopulation (late apoptotic cells) did not significantly change. These data indicated that $\mathrm{HG}$ inhibited the apoptosis of VSMCs and that HJT significantly increased the apoptosis (mainly early apoptosis) of VSMCs.

\section{Experimental Validation of Key Targets}

The AKT pathway is often overactivated by $\mathrm{HG}^{18}$ and is associated with cell proliferation, migration, and apoptosis. To delineate the response of VSMCs under stimulation with HG, the phosphorylation of AKT (pAKT) was examined. HG significantly increased the pAKT/tAKT ratio $(\mathrm{P}<0.01$ vs MG, Figure 7). HJT treatment significantly reduced the pAKT/tAKT ratio $(\mathrm{P}<0.05$ vs HG). MMP9 and PCNA, the downstream factors of AKT in regulating cell migration and proliferation, were significantly upregulated by HG, while HJT suppressed the expression of MMP9 and PCNA at concentrations of 20 and $40 \mathrm{~mL} / \mathrm{L}(\mathrm{P}<0.05$ vs $\mathrm{HG})$.

The inactivation of AKT induces p53 activation, causing alterations in Bcl-2 family proteins and caspase activation, which contribute to the proapoptotic signal. ${ }^{19}$ The results indicated that $\mathrm{HG}$ reduced $\mathrm{p} 53$ activation, cleaved caspase-3 levels and the $\mathrm{Bax} / \mathrm{Bcl}-2$ ratio compared with those of the MG group $(P<0.05$, Figure 7$)$. HJT at concentrations of $40 \mathrm{~mL} / \mathrm{L}$ significantly increased the levels of p53 and cleaved caspase-3 and the Bax/Bcl-2 ratio $(P<0.01$ vs HG).

\section{HJT Reversed HG-Induced Changes in VSMCs Partially Through the AKT Pathway}

To investigate the potential mechanism of HJT in HG-induced VSMCs, the AKT pathway was evaluated. After incubation 
A

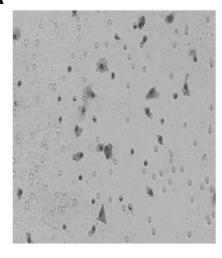

MG

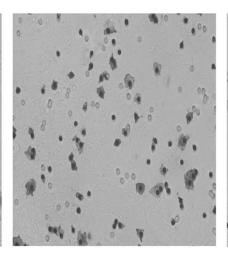

HG

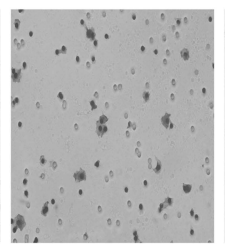

HG+HJT10

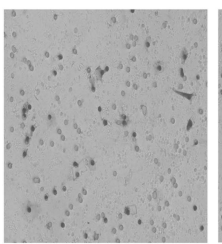

HG+HJT20

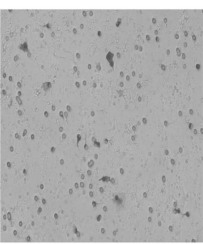

HG+HJT40

B

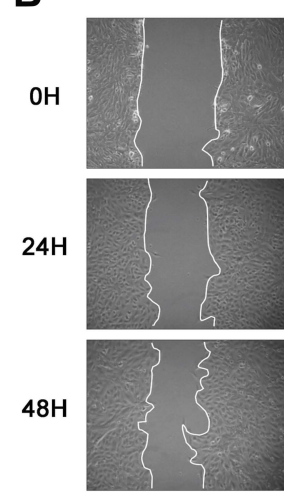

MG
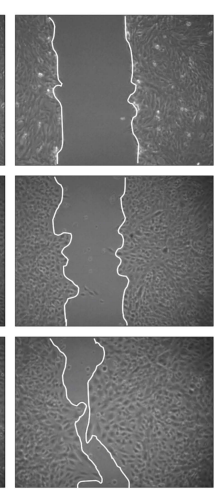

HG
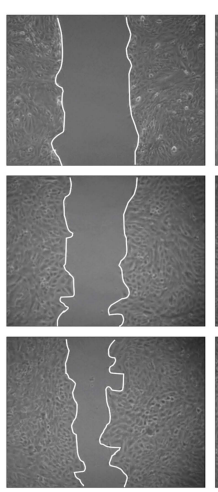

HG+HJT10

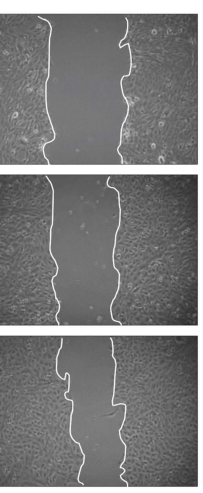

HG+HJT20

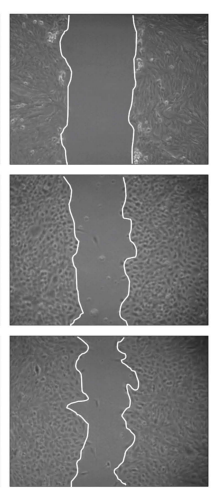

HG+HJT40
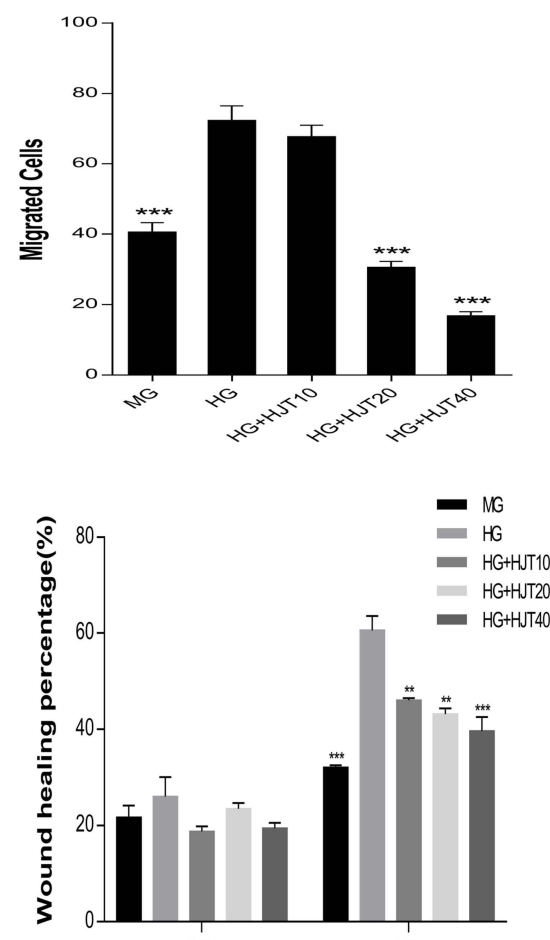

$24 \mathrm{H}$

$48 \mathrm{H}$

Figure 5 HJT suppressed the HG-induced migration of VSMCs. (A). Left: Transwell assay. Right: Quantitation of migrated VSMCs; (B). Left: Wound-healing assays at 0 hr, $24 \mathrm{hrs}$ and $48 \mathrm{hrs}$ after wounding. Right: Quantification of the wound healing percentage. Data were presented by mean \pm SD. **p<0.0 I, ***p $<0.00 \mathrm{I}$ all compared with $\mathrm{HG}$ group.

Abbreviations: HJT, Hongjingtian Injection; MG, mannitol group; HG, high glucose; HJT I0, IOml/L HJT; HJT 20, 20ml/L HJT; HJT 40, 40ml/L HJT.

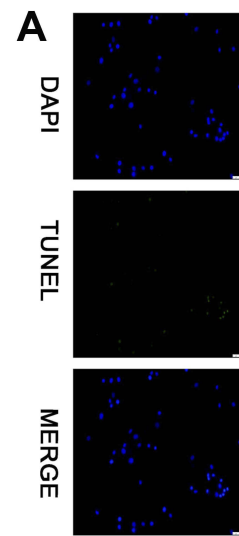

B

B PI

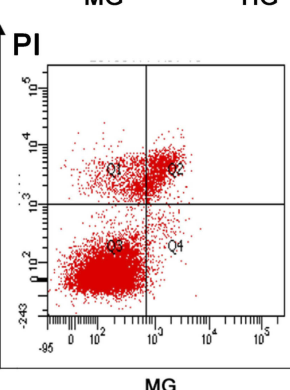

HG
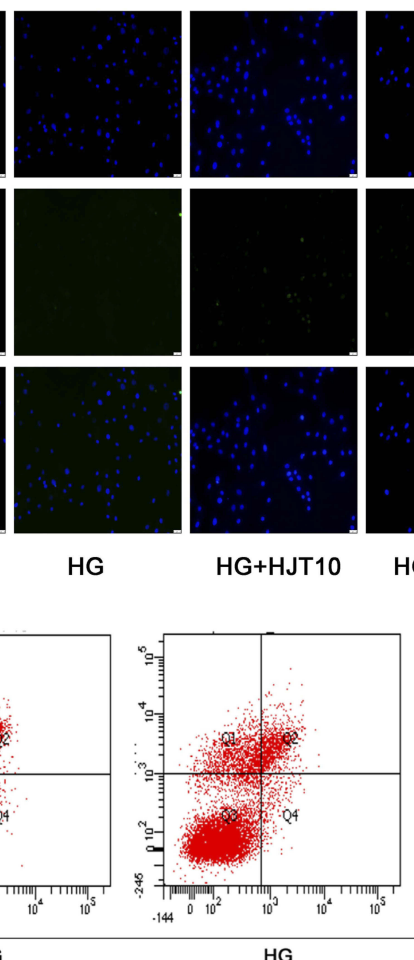
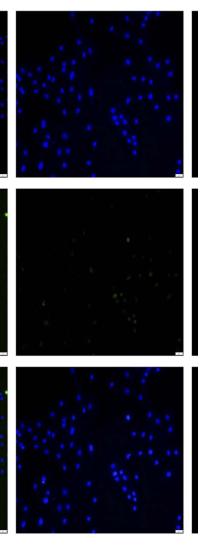

HG+HJT10
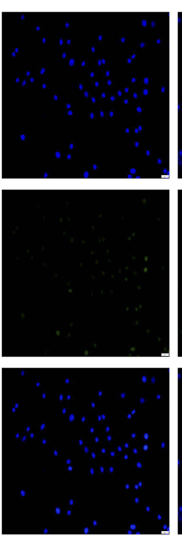

HG+HJT20
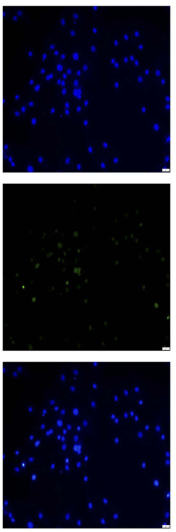

HG+HJT40

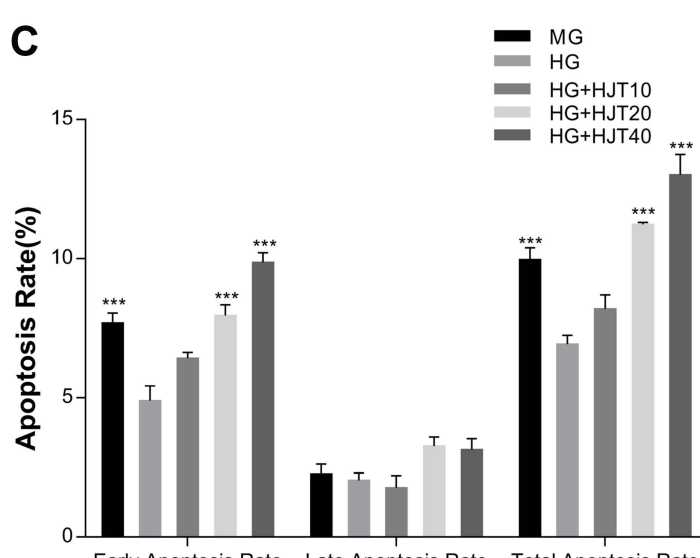

Early Apoptosis Rate Late Apoptosis Rate Total Apoptosis Rate
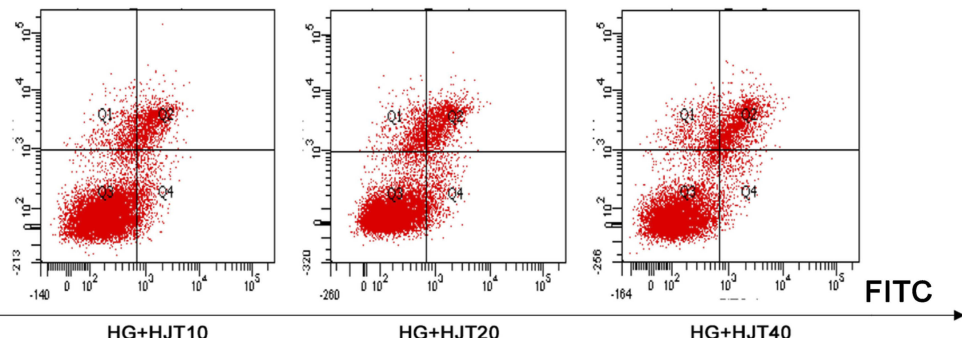

Figure 6 HJT reversed the apoptosis resistance of VSMCs in the treatment of HG. (A). Representative images of TUNEL staining. The bar size is $25 \mu$ m. (B). Annexin V-FITC/PI apoptosis assay. (C). Quantification of the apoptosis rate. Values were mean \pm SD. $* * * p<0.00 I$ vs HG group.

Abbreviations: DAPI, 4',6-diamidino-2-phenylindole; TUNEL, terminal deoxynucleotidyl transferase dUTP nick end labeling; HJT, Hongjingtian Injection; MG, mannitol group; HG, high glucose; HJT I0, $10 \mathrm{ml} / \mathrm{L} \mathrm{HJT;} \mathrm{HJT} \mathrm{20,} \mathrm{20ml/L} \mathrm{HJT;} \mathrm{HJT} \mathrm{40,} \mathrm{40ml/L} \mathrm{HJT.}$ 
A

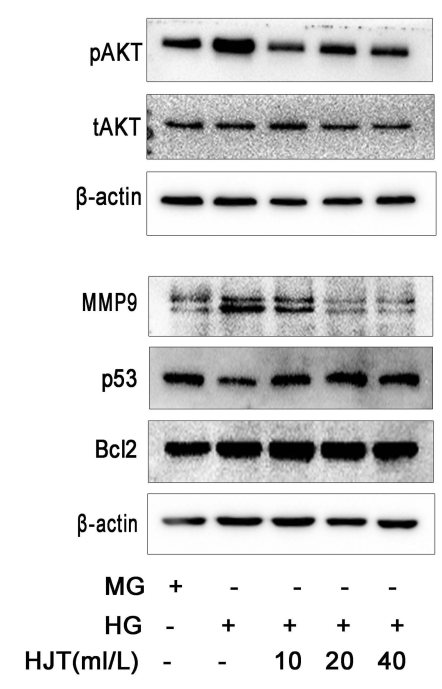

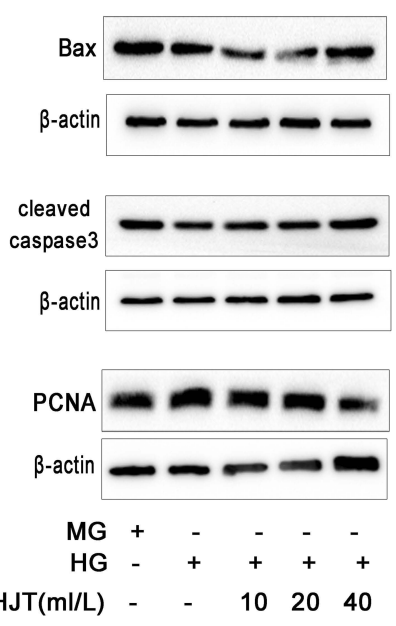

B

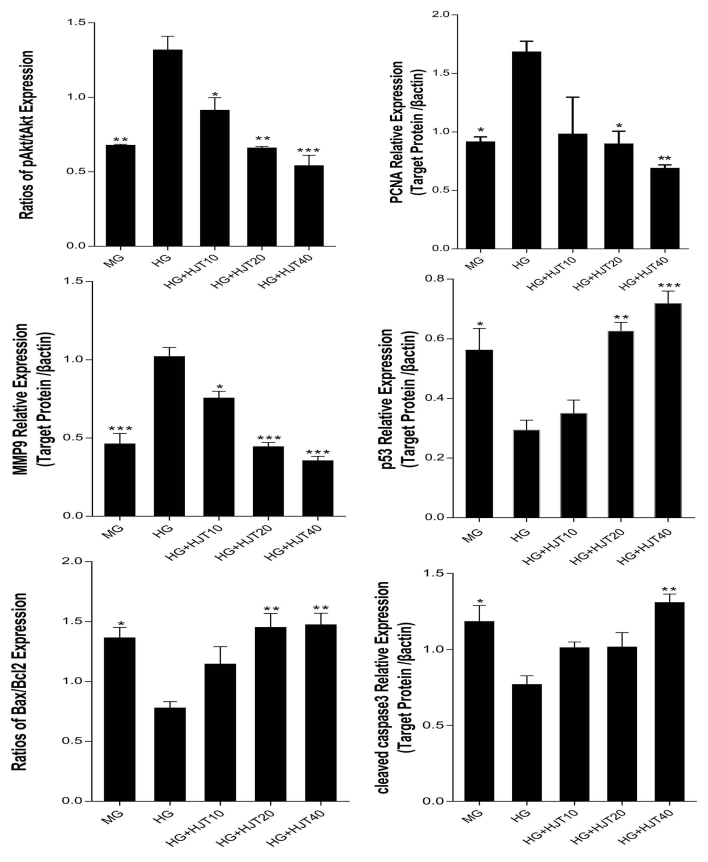

Figure 7 Western blotting studies. (A). Western blotting analysis of pAKT, tAKT, PCNA, MMP9, Bcl-2, Bax, p53, cleaved caspase-3 with $\boldsymbol{\beta}$-actin as an internal reference. (B). Quantification of the Western blotting results. Data was presented as the mean $\pm \mathrm{SD}$. ${ }^{*} \mathrm{p}<0.05,{ }^{*} \mathrm{p}<0.0 \mathrm{I}$, *** $<<0.00 \mathrm{I}$ all compared with $\mathrm{HG}$ group.

Abbreviations: p, phosphorylated; t, total; HJT, Hongjingtian Injection; MG, mannitol group; HG, high glucose; HJT I0, 10ml/L HJT; HJT 20, 20ml/L HJT; HJT 40, 40ml/L HJT.

with SC79 for $48 \mathrm{hrs,}$ as shown in Figure 8A, HG-induced cells treated with SC79 had significantly increased the expression of pAKT and MMP9, and reduced the level of cleaved caspase-3 and the Bax/Bcl-2 ratio compared with $\mathrm{HG}$. Furthermore, the effects of HJT40 on VSMCs were partially reversed by treatment with SC79. We then measured the protein level of key targets under LY294002 stimulation. AKT phosphorylation was markedly decreased compared to that of the HG group $(\mathrm{P}<0.05$, Figure $8 \mathrm{~B})$ and was accompanied by reduced expression of MMP9 and PCNA and increased expression of $\mathrm{p} 53$, cleaved caspase-3 and Bax/Bcl2 ratio. These effects of HG with LY294002 were similar to those of $\mathrm{HG}$ with $40 \mathrm{~mL} / \mathrm{L}$ HJT. These results demonstrated that HJT could suppress proliferation and migration, and promote apoptosis of VSMCs through the AKT pathway, confirming the network analysis findings.

\section{Discussion}

Accumulating evidence has suggested that $\mathrm{HG}$ alters the balance of VSMC proliferation and apoptosis, stimulates proliferation and migration, and inhibits apoptosis, ${ }^{20}$ which implies it could be one of the causes of DA. ${ }^{21}$ VSMCs are involved in the pathogenesis of atherosclerosis by accelerating proliferation and migration toward the lesion, extracellular matrix generation and plaque progression. ${ }^{22}$ Therefore, targeting VSMCs might be an effective strategy for treating diabetic vascular complications.

HJT has been applied for vascular disease therapy for many years. ${ }^{23}$ Recent studies have revealed that five components of $\mathrm{HJT}^{11}$ possess multiple pharmacological effects. Salidroside, one of the active compounds of HJT, exerted remarkable inhibitory effects on VSMC proliferation. ${ }^{24}$ According to Yang et $\mathrm{al}^{25}$ gallic acid attenuated the proliferation of human umbilical artery smooth muscle cells (HUASMCs). Tyrosol may be an effective drug in preventing atherosclerotic processes. ${ }^{26}$ Behrangi $\mathrm{N}$ also reported that p-hydroxycinnamic acid inhibited the gene expression of MMP9 in rats. ${ }^{27}$ However, there has been no scientific research on the synergistic effects of HJT on VSMCs to our knowledge.

Considering the multicomponent and multitarget characteristics of HJT, a network pharmacology approach ${ }^{28,29}$ was used to investigate the underlying mechanism of HJT for DA therapy. It was predicted that HJT could regulate cell proliferation, apoptosis, and migration processes via the AKT pathway by directly interacting the targets $A K T$, $M M P 9, C A S P 3, T P 53, B C L 2$ and $B A X$. Based on the results of network pharmacology analysis, cell experiments were used to validate the predicted mechanisms. Consistent with previous findings, our results showed that $\mathrm{HG}$ promoted proliferation and migration, but 
A

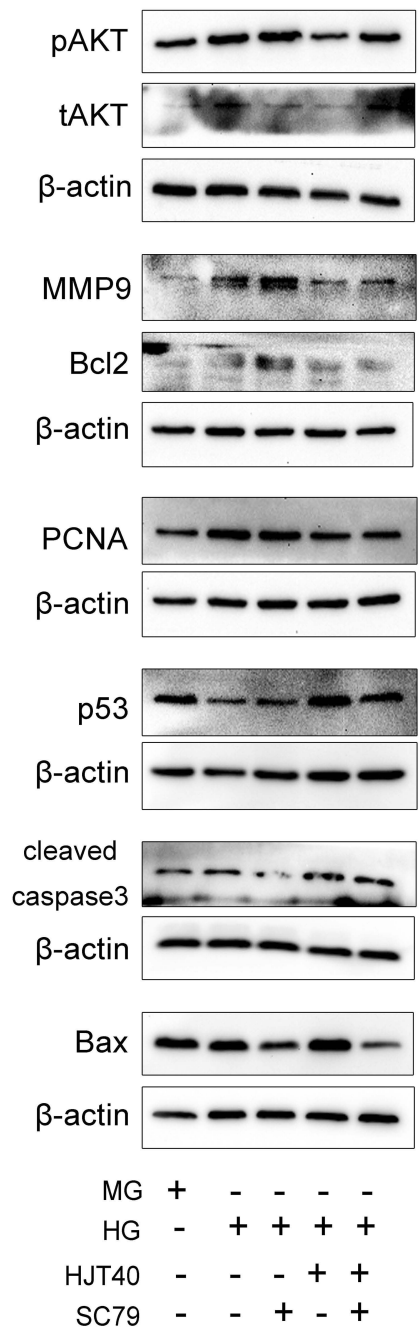

B
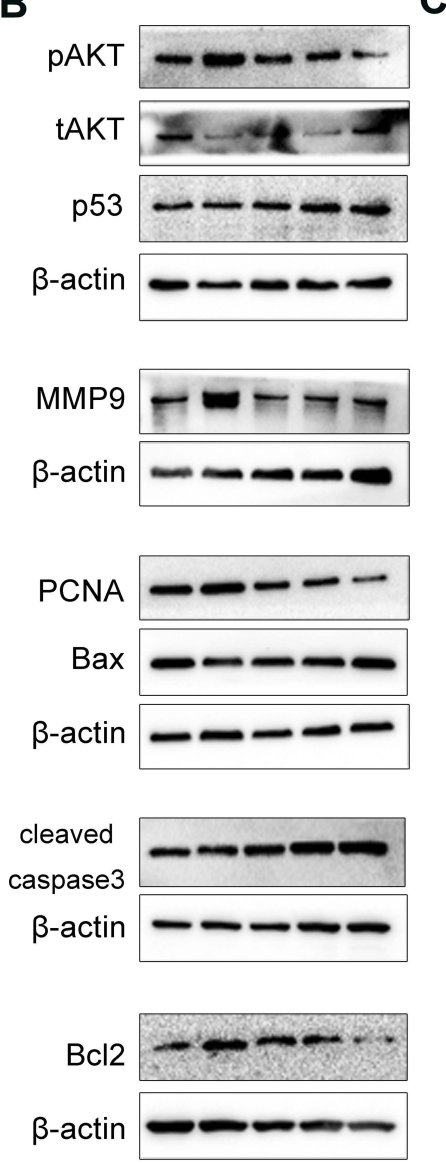

$\mathrm{MG}+-\quad-$

$\mathrm{HG}-++++$

HJT40 - --++

LY294002 - - + - +
C
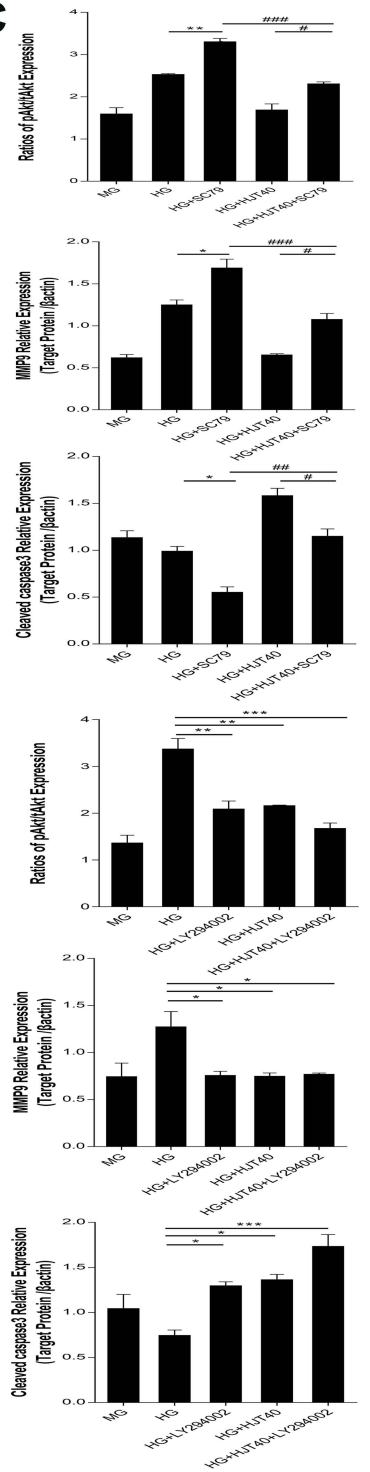
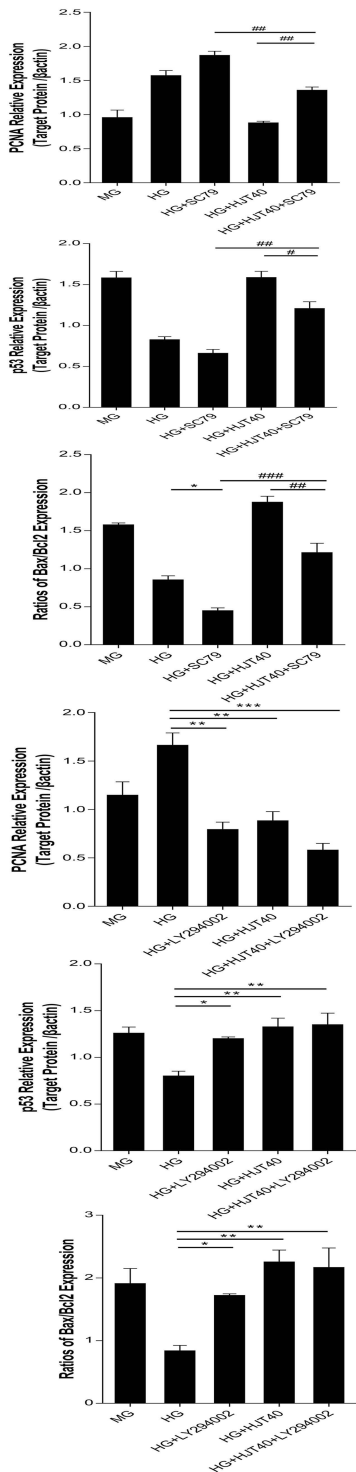

Figure 8 (A). SC79 can reverse the inhibitory effects of HJT on HG-induced VSMCs. (B). LY294002 can inhibit the HG-induced changes in VSMCs consistent with the effects of HJT. (C). Quantification of the Western blotting results. Data was presented as the mean \pm SD. ${ }^{*} \mathrm{p}<0.05$, ${ }^{* *} \mathrm{p}<0.0 \mathrm{I}$, ${ }^{* * *} \mathrm{p}<0.00 \mathrm{I}$ in comparison with HG group, $\# p<0.05, \# \mathrm{p}<0.01, \# \# \mathrm{p}<0.001$ in comparison with HG+HJT40+SC79 group.

Abbreviations: p, phosphorylated; t, total; HJT, Hongjingtian Injection; MG, mannitol group; HG, high glucose; HJT 40, 40mI/L HJT.

inhibited apoptosis of VSMCs. However, these HGstimulated changes were significantly reversed by HJT. In addition, we investigated the underlying mechanism further.

AKT, an important factor in PI3K-initiated signal transduction pathways, is tightly related to cell survival and cell death. ${ }^{30}$ Previous research reported that the AKT pathway participated in HG-induced VSMC proliferation, migration and apoptosis. ${ }^{20}$ Aberrantly activated AKT upregulated the expression of PCNA and MMP9, ${ }^{31}$ but downregulated the expression of $\mathrm{p} 53 .^{32}$ The downstream factor MMP9, one of the essential components of matrix metalloproteinases, is involved in cell adhesion and migration. ${ }^{33}$ As shown in our data, HJT suppressed the levels of pAKT, MMP9 and PCNA in HG-induced VSMCs.

Inhibition of AKT activity, in turn, activates p53 and sequentially stimulates mitochondria-dependent apoptosis pathways. $^{34}$ The tumor suppressor p53 functions as a transcriptional factor to regulate the downstream Bcl-2 family. ${ }^{35}$ The apoptosis-promoting protein Bax and antiapoptotic protein $\mathrm{Bcl}-2$ are two of the most critical Bcl-2 family members. ${ }^{36}$ The $\mathrm{Bax} / \mathrm{Bcl}-2$ ratio is an index of apoptosis, and its increase induces the activation of caspases-3 and ultimately results in apoptosis. ${ }^{37}$ In this study, 
HG decreased the p53, cleaved caspase-3, and Bax/Bcl-2 ratio at the protein level. However, upregulation of $\mathrm{p} 53$ and caspase- 3 expression and increase in the Bax/Bcl-2 ratio was observed following HJT treatment in VSMCs.

To investigate whether AKT pathways are involved in the effects of HJT on HG-induced changes in VSMCs, an activator and inhibitor of AKT were used. In this work, we found that HJT in combination with LY294002 had generally consistent inhibitory effects on HG-induced VSMCs. Similarly, SC79, a strong AKT agonist, partially reversed the neutralizing effects of HJT. These results indicated that HJT suppressed cell proliferation, migration and induced apoptosis partly through the AKT pathway.

Our study has limitations. Although VSMCs play an important role in vascular disease, we do not know whether the therapeutic action of HJT on DA is cell-type specific. Moreover, the suppression of proliferation and migration and the promotion of apoptosis in VSMCs by HJT was limited to the AKT pathway. However, other signaling pathways have also been reported in DA. These interesting questions require further investigation.

\section{Conclusion}

In summary, this research systematically illustrated for the first time the mechanisms of action of HJT for DA therapy. HJT exhibited antiproliferative, antimigratory and proapoptotic effects on HG-induced VSMCs partly by the inhibiting AKT pathway. These results may provide data support to support the clinical application of HJT for DA therapy.

\section{Acknowledgment}

This work was supported by the National Natural Science Foundation of China (81570742) and the Development of Science and Technology of Shandong Traditional Chinese Medicine (No. 2017-172).

\section{Disclosure}

The authors report no conflicts of interest in this work.

\section{References}

1. Cho NH, Shaw JE, Karuranga S, et al. IDF diabetes atlas: global estimates of diabetes prevalence for 2017 and projections for 2045 Diabetes Res Clin Pract. 2018;138:271-281. doi:10.1016/j.diabres. 2018.02.023

2. Maric-Bilkan C. Sex differences in micro- and macro-vascular complications of diabetes mellitus. Clin Sci (Lond). 2017;131(9):833-846. doi:10.1042/CS20160998

3. Katakami N. Mechanism of development of atherosclerosis and cardiovascular disease in diabetes mellitus. J Atheroscler Thromb. 2018;25 (1):27-39. doi:10.5551/jat.RV17014
4. Shi N, Chen SY. Smooth muscle cell differentiation: model systems, regulatory mechanisms, and vascular diseases. $J$ Cell Physiol. 2016;231(4):777-787. doi:10.1002/jcp.25208

5. Allen DA, Yaqoob MM, Harwood SM. Mechanisms of high glucose-induced apoptosis and its relationship to diabetic complications. J Nutr Biochem. 2005;16(12):705-713. doi:10.1016/ j.jnutbio.2005.06.007

6. Rudijanto A. The role of vascular smooth muscle cells on the pathogenesis of atherosclerosis. Acta Med Indones. 2007;39(2):7.

7. Shi H, Wang D, Zhao D, Liu G. Study on the influence of serum hs-CRP and AS in patients with Primary Hypertension with Sofren injection. Cardiovasc Dis J Integr Tradit Chin West Med. 2015;3(10):3.

8. Zhao J, Liao Y, Chen S. Effect of Dazhu Hongjingtian injection on hemorrheology in type 2 diabetes. Clini J Chin Med. 2016;8:2.

9. Ma YM, Zhang XZ, Su ZZ, et al. Insight into the molecular mechanism of a herbal injection by integrating network pharmacology and in vitro. J Ethnopharmacol. 2015;173:91-99. doi:10.1016/j.jep.2015.07.016

10. Liu Y, Chen C, Qiu J, et al. Characterization of the chemical constituents in Hongjingtian injection by liquid chromatography quadrupole time-of-flight mass spectrometry. Biomed Chromatogr. 2019;33 (3):e4446. doi:10.1002/bmc.v33.3

11. Yang S, Zhao G, Wang R, et al. Study on fingerprint of Hongjingtian injection by HPLC. J Tianjin Med Univ. 2016;22:164-167.

12. Wang L, Zhang Z, Liang L, Wu Y, Zhong J, Sun X. Anti-high mobility group box-1 antibody attenuated vascular smooth muscle cell phenotypic switching and vascular remodelling after subarachnoid haemorrhage in rats. Neurosci Lett. 2019;708:134338. doi:10.1016/j.neulet.2019.134338

13. He X, Jiang $\mathrm{H}$, Gao $\mathrm{F}$, Liang $\mathrm{S}$, Wei $M$, Chen L. Indoxyl sulfate-induced calcification of vascular smooth muscle cells via the PI3K/Akt/NF-kappaB signaling pathway. Microsc Res Tech. 2019. doi:10.1002/jemt.23369

14. Han Y, Jiang Q, Wang Y, et al. The anti-proliferative effects of oleanolic acid on A7r5 cells-Role of UCP2 and downstream FGF-2/ p53/TSP-1. Cell Biol Int. 2017;41(12):1296-1306. doi:10.1002/cbin. v41.12

15. Zhang S, Zhang L, Zhang H, et al. Hongjingtian injection attenuates myocardial oxidative damage via promoting autophagy and inhibiting apoptosis. Oxid Med Cell Longev. 2017;2017:6965739. doi:10.1155/ 2017/6965739

16. Liu Y, Li X, Jiang S, Ge Q. Tetramethylpyrazine protects against high glucose-induced vascular smooth muscle cell injury through inhibiting the phosphorylation of JNK, p38MAPK, and ERK. $J$ Int Med Res. 2018;46(8):3318-3326. doi:10.1177/0300060518781705

17. Xing X, Chen S, Li L, et al. The active components of Fuzheng Huayu formula and their potential mechanism of action in inhibiting the hepatic stellate cells viability - a network pharmacology and transcriptomics approach. Front Pharmacol. 2018;9:525. doi:10.3389/fphar.2018.00525

18. Cai W, Zhang J, Yang J, et al. MicroRNA-24 attenuates vascular remodeling in diabetic rats through $\mathrm{PI} 3 \mathrm{~K} / \mathrm{Akt}$ signaling pathway. Nutr Metab Cardiovasc Dis. 2019;29(6):11. doi:10.1016/j.numecd. 2019.03.002

19. Manfe V, Biskup E, Rosbjerg A, et al. miR-122 regulates p53/Akt signalling and the chemotherapy-induced apoptosis in cutaneous T-cell lymphoma. PLoS One. 2012;7(1):e29541. doi:10.1371/journal.pone.0029541

20. Shi L, Ji Y, Jiang X, et al. Liraglutide attenuates high glucose-induced abnormal cell migration, proliferation, and apoptosis of vascular smooth muscle cells by activating the GLP-1 receptor, and inhibiting ERK1/2 and PI3K/Akt signaling pathways. Cardiovasc Diabetol. 2015;14:18. doi:10.1186/s12933-015-0177-4

21. Wang K, Deng X, Shen Z, et al. High glucose promotes vascular smooth muscle cell proliferation by upregulating proto-oncogene serine/threonine-protein kinase Pim-1 expression. Oncotarget. 2017;8(51):88320-88331. doi:10.18632/oncotarget.19368 
22. Baumer Y, McCurdy S, Alcala M, et al. CD98 regulates vascular smooth muscle cell proliferation in atherosclerosis. Atherosclerosis. 2017;256:105-114. doi:10.1016/j.atherosclerosis.2016.11.017

23. Sun X, Liu Y, Jiang D, Chen F, Xie Y, Sun Y. Research progress on the pharmacological effects of Rhodiola rosea. Acta Chin Med Pharmacol. 2017;45(6):4.

24. Zhuang X, Maimaitijiang A, Li Y, Shi H, Jiang X. Salidroside inhibits high-glucose induced proliferation of vascular smooth muscle cells via inhibiting mitochondrial fission and oxidative stress. Exp Ther Med. 2017;14(1):515-524. doi:10.3892/etm.2017.4541

25. Yang Z, Xiong K, Qi P, et al. Gallic acid tailoring surface functionalities of plasma-polymerized allylamine-coated $316 \mathrm{~L}$ SS to selectively direct vascular endothelial and smooth muscle cell fate for enhanced endothelialization. ACS Appl Mater Interfaces. 2014;6 (4):2647-2656. doi:10.1021/am405124z

26. Vivancos M, Moreno JJ. Effect of resveratrol, tyrosol and beta-sitosterol on oxidised low-density lipoprotein-stimulated oxidative stress, arachidonic acid release and prostaglandin E2 synthesis by RAW 264.7 macrophages. Br J Nutr. 2008;99(6):1199-1207. doi:10.1017/S0007114507876203

27. Behrangi N, Namvar N, Ataei M, Dizaji S, Javdani G, MH S. MMP9 gene expression variation by ingesting tart cherry and P-coumaric acid during remyelination in the cuprizone mouse model. Acta Med Iran. 2017;55(9):10.

28. Gao L, Wang KX, Zhou YZ, Fang JS, Qin XM, Du GH. Uncovering the anticancer mechanism of Compound Kushen Injection against HCC by integrating quantitative analysis, network analysis and experimental validation. Sci Rep. 2018;8(1):624. doi:10.1038/s41598-017-18325-7

29. Lee AY, Park W, Kang TW, Cha MH, Chun JM. Network pharmacology-based prediction of active compounds and molecular targets in Yijin-Tang acting on hyperlipidaemia and atherosclerosis. J Ethnopharmacol. 2018;221:151-159. doi:10.1016/j.jep.2018.04.027
30. Epand RM. Features of the phosphatidylinositol cycle and its role in signal transduction. J Membr Biol. 2017;250(4):353-366. doi:10.1007/ s00232-016-9909-y

31. Zhang C-Y, Yu M-S, Li X, Zhang Z, Han C-R, Yan B. Overexpression of long non-coding RNA MEG3 suppresses breast cancer cell proliferation, invasion, and angiogenesis through AKT pathway. Tumor Biol. 2017;39(6):1010428317701311.

32. Xu W, Gao L, Li T, Zheng J, Shao A, Zhang J. Mesencephalic Astrocyte-Derived Neurotrophic Factor (MANF) protects against neuronal apoptosis via activation of Akt/MDM2/p53 signaling pathway in a rat model of intracerebral hemorrhage. Front Mol Neurosci. 2018;11. doi:10.3389/fnmol.2018.00176

33. Newby AC. Matrix metalloproteinases regulate migration, proliferation, and death of vascular smooth muscle cells by degrading matrix and non-matrix substrates. Cardiovasc Res. 2006;69(3):614-624. doi:10.1016/j.cardiores.2005.08.002

34. Shankar S, Srivastava RK. Involvement of Bcl-2 family members, phosphatidylinositol 3'-kinase/AKT and mitochondrial p53 in curcumin (diferulolylmethane)-induced apoptosis in prostate cancer. Int J Oncol. 2007;30(4):905-918.

35. Vousden KH, Lu X. Live or let die: the cell's response to p53. Nat Rev Cancer. 2002;2(8):594-604. doi:10.1038/nrc864

36. Tait SW, Green DR. Mitochondrial regulation of cell death. Cold Spring Harb Perspect Biol. 2013;5(9):a008706-a008706. doi:10.11 01/cshperspect.a008706

37. Ashkenazi A, Dixit VM. Death receptors: signaling and modulation. Science. 1998;281(5381):1305-1308. doi:10.1126/science.281.5381.13 05

\section{Publish your work in this journal}

Drug Design, Development and Therapy is an international, peerreviewed open-access journal that spans the spectrum of drug design and development through to clinical applications. Clinical outcomes, patient safety, and programs for the development and effective, safe, and sustained use of medicines are a feature of the journal, which has also been accepted for indexing on PubMed Central. The manuscript management system is completely online and includes a very quick and fair peer-review system, which is all easy to use. Visit http://www. dovepress.com/testimonials.php to read real quotes from published authors. 